Please do not remove this page

RMIT

UNIVERSITY

\title{
Low-cost RPAS navigation and guidance system using Square Root Unscented Kalman Filter
}

Cappello, Francesco; Ramasamy, Subramanian; Sabatini, Roberto

https://researchrepository.rmit.edu.au/esploro/outputs/9921862648301341/filesAndLinks?institution=61 RMIT_INST\&index=null

Cappello, F., Ramasamy, S., \& Sabatini, R. (2015). Low-cost RPAS navigation and guidance system using Square Root Unscented Kalman Filter. Proceedings of the SAE 2015 AeroTech Congress and Exhibition, 1-11. https://doi.org/10.4271/2015-01-2459

Document Version: Accepted Manuscript

Published Version: https://doi.org/10.4271/2015-01-2459

Repository homepage: https://researchrepository.rmit.edu.au

(C) 2015 SAE International

Downloaded On 2023/04/26 12:06:04 +1000

Please do not remove this page 
Thank you for downloading this document from the RMIT Research Repository.

The RMIT Research Repository is an open access database showcasing the research outputs of RMIT University researchers.

RMIT Research Repository: http://researchbank.rmit.edu.au/

\section{Citation:}

Cappello, F, Ramasamy, S and Sabatini, R 2015, 'Low-cost RPAS navigation and guidance system using Square Root Unscented Kalman Filter', in Proceedings of the SAE 2015

AeroTech Congress and Exhibition, Warrendale, PA, United States, 22-24 September 2015, pp. 1-11.

See this record in the RMIT Research Repository at:

https://researchbank.rmit.edu.au/view/rmit:33427

Version: Accepted Manuscript

Copyright Statement: (c) 2015 SAE International

Link to Published Version:

http://dx.doi.org/10.4271/2015-01-2459 


\title{
Low-Cost RPAS Navigation and Guidance System using Square Root Unscented Kalman Filter
}

\author{
Francesco Cappello, Subramanian Ramasamy, Roberto Sabatini \\ RMIT University - SAMME, Melbourne, Australia
}

Copyright $\odot 2015$ SAE International

\begin{abstract}
Multi-Sensor Data Fusion (MSDF) techniques involving satellite and inertial-based sensors are widely adopted to improve the navigation solution of a number of mission- and safety-critical tasks. Current Navigation and Guidance Systems (NGS) employing MSDF algorithms do not meet the required level of performance in all flight phases of small Remotely Piloted Aircraft Systems (RPAS). Hence in order to satisfy the Required Navigation Performance (RNP), an innovative Square Root-Unscented Kalman Filter (SR-UKF) based NGS is implemented and compared with a conventional UKF design. The presented NGS architectures employ a number of state-of-the-art low-cost sensors including; Global Navigation Satellite Systems (GNSS), Micro-Electro-Mechanical System (MEMS) based Inertial Measurement Unit (IMU) and Vision Based Navigation (VBN) sensors. Additionally, an Aircraft Dynamics Model (ADM), which is essentially a knowledge based module, is employed to compensate for the MEMS-IMU sensor shortcomings in high-dynamics attitude determination tasks. The ADM acts as a virtual sensor and its measurements are processed with non-linear estimation techniques in order to increase the operational validity time. An improvement in the ADM navigation state vector (i.e., position, velocity and attitude) measurements is obtained, thanks to the accurate modeling of aircraft dynamics and advanced processing techniques. A novel SR-UKF based VBN-IMU-GNSS-ADM (SR-U-VIGA) architecture design is implemented and compared with a conventional UKF based design (U-VIGA) in a small RPAS (AEROSONDE) integration scheme exploring a representative cross-section of the operational flight envelope. The comparison of the state vector demonstrates the capability of SR-U-VIGA and U-VIGA systems to fulfill the relevant RNP criteria, including precision approach tasks. Furthermore, the computation time of SR-U-VIGA system is lower when compared to U-VIGA NGS allowing for an enhanced implementation in real-time applications.
\end{abstract}

\section{Introduction}

The scientific community of the aerospace industry is currently focusing on developing innovative systems to integrate Remotely Piloted Aircraft System (RPAS) into the current and future classes of airspace. The integration is dependent on advanced Communication, Navigation and Surveillance (CNS) technologies in order to meet the Required Navigation Performance (RNP) levels [1-3]. Current civil and military RPAS operations are mostly segregated and the challenges involved with their integration into commercial airspace are currently being addressed through various operational and technological developments. The focus areas of development are concentrated on enhanced CNS and Air Traffic Management (ATM) $(\mathrm{CNS} / \mathrm{ATM})$ and Avionics $(\mathrm{CNS}+\mathrm{A})$ solutions, Detect-and-Avoid (DAA) solutions and continuous airworthiness aspects. To realise global harmonization of RPAS, a roadmap is envisaged by the International Civil Aviation Organization (ICAO) as part of its Aviation System Block Upgrades (ASBU) plan [4]. Basic procedures and functions, which will initiate integration, include both cooperative and non-cooperative DAA functions. Secure and safe Line-of-Sight (LOS) and Beyond-Line-of-Sight (BLOS) communication links are essential for RPAS to maintain continuous contact with the Ground Control Station (GCS). Significant outcomes are also expected from findings of large-scale and regional ATM modernization programs including Single European Sky ATM Research (SESAR) and Next Generation Air Transportation System (NextGen). The key enabling technologies required for the evolution of CNS+A framework have been identified as part of these programmes [5-10]. The requirements for RPAS to be integrated into all classes of airspace are illustrated in Figure 1.

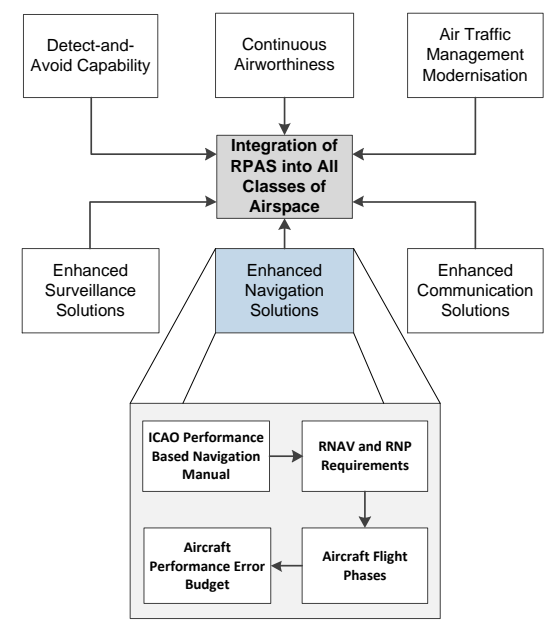

Figure 1. Requirements for RPAS integration into all classes of airspace.

Enhanced surveillance solutions based on Automatic Dependent Surveillance-Broadcast (ADS-B) system and Traffic Collision Avoidance System (TCAS) are required for addressing cooperative DAA functions [11-18]. The Performance Based Navigation (PBN) concept specifies that aircraft RNP and area navigation (RNAV) requirements have to be met for all flight phases [11]. These navigation specifications are defined at a sufficient level of detail in order to facilitate global harmonization by providing specific implementation guidance for national aviation regulators and 
operators. The RNP is in turn translated to technical requirements, which aid in the determination of specific airborne sensors that can be employed onboard the RPAS [19, 20]. The sensor requirements dictate the following: physical characteristics of the sensors including size, weight and volume, support requirements such as electrical power, accuracy and precision [20].

High-integrity based integrated Navigation and Guidance Systems (NGS) are required to meet the RNP criteria that are dictated by the International Civil Aviation Organization (ICAO) [19]. Current NGS are not capable of achieving the required level of performance in all flight phases of a small RPAS. In this research, the implementation of NGS is based on low-cost avionic sensors, which provide a number of benefits including reduction in weight and volume, as well as satisfying support requirements in an inexpensive approach [20]. The appropriate combination of sensors and integration algorithms enable the NGS to meet accuracy, continuity, availability and integrity requirements set by ICAO [4]

Global Navigation Satellite System (GNSS) and Micro-ElectroMechanical System (MEMS) based Inertial Measuring Unit (IMU) are a highly synergistic combination of navigation sensors capable of providing an accurate navigation state vector better than any standalone sensor. Vision Based Navigation (VBN) sensors are typically adopted for precision approach and landing (i.e., the most demanding and potentially safety-critical flight phase). Aircraft Dynamics Models (ADM) are not currently employed in any commercial systems as a virtual sensor, however previous research $[19,20,22$, 23 ] shows that it can act as a knowledge-based module augmenting the navigation state vector and thus increasing the accuracy of the navigation solution $[19,20,22,23]$. The performance of the ADM is satisfactory only for a short time period and hence requires reinitialisation. Therefore to increase the operational potential of the ADM, approximate Bayesian estimation techniques are adopted to process the aircraft dynamics (i.e., aircraft trajectory and attitude motion). The ADM is represented by non-linear ordinary differential equations, using which the aerodynamic forces and moments acting on the RPAS are estimated.

\section{Multi-Sensor Data Fusion}

Inertial based navigation using Kalman filtering has been the de facto methodology used in aeronautical navigation for more than thirty years [24]. Both absolute (Global Positioning System (GPS), radars, etc.) and relative (odometers, IMUs, etc.) position sensors provide noisy measurements and hence in most cases, filtering techniques are essential [24]. Data fusion by definition is the integration process that involves multiple knowledge, data or information sources representing the same real-world object to provide a consistent, accurate, and useful representation [25]. Multi-Sensor Data Fusion (MSDF) is the process of combining observations from a number of different sensors to provide a robust and complete description of an environment or process of interest where large volumes of data is implemented by combining information from multiple sensors to achieve results that are not possible from a single sensor or information source [26]. MSDF is different from single sensor estimation in the aspect that data fusion combines multiple sensor information to provide the best possible state estimation, while single sensor estimation utilizes sensor characteristics in order to improve the overall performance of that sensor. Data fusion was primarily used in statistics and estimation fields and then later featured more often in robotic applications to increase agent/platform autonomy. Robotic systems such as RPAS do not have an on-board pilot and therefore rely on sensors and systems to perform the planned missions. Therefore methods that improve the accuracy of obtained measurements are imperative to the increase in overall navigation performance. Physical agents such as RPAS are inherently unstable, due to factors including;

- Environmental stochastic unpredictability

- Sensor limited noise and

- Model inaccuracies and errors

To obtain an optimal solution (i.e., optimal state vector measurement), the traditional Kalman Filter (KF) is typically applied. A KF being an optimal estimator is recursive by nature and in order for new measurements to be processed in real time it infers parameters of interest from indirect and uncertain observations. The $\mathrm{KF}$ is derived on the assumption that the noises are normally distributed, therefore a likelihood function of the prediction error can be calculated, which results in estimation of the state vector. When presented with a non-linear function, linear filtering techniques are not capable to provide an accurate solution. In realistic scenarios involving maneuvering tasks, the motions are rarely linear and therefore approximation techniques are required. The Extended Kalman Filter (EKF) has been the most commonly used data fusion technique in numerous non-linear estimation applications. During these last thirty years, different architectures for navigation data integration were deployed and investigated, using a number of algorithms in filtering theory. Both linear and non-linear filters were applied to estimate different state navigation compounds as Position, Velocity and Attitude (PVA) of the vehicles [27]. Recent advancements in the application of Bayesian inference and non-linear approximation algorithms are largely attributed to the availability of processors with greater computational capabilities. Approximate estimation techniques are used to predict and correct the new state information based on previous measurements. Specifically, a proportional term is used and hence the prediction error is statistically minimized allowing for a more accurate estimation of the state vector [28]. Although the EKF is one of the most widely used filtering methods for non-linear system estimation, this filter has two key drawbacks that make it challenging for a practical implementation [28-35] including:

- It does not take into account probabilistic uncertainty of the random variables of the system state and noise when the linearization of the system equations is performed and

- The accuracy of the propagated mean and the covariance is limited to the first order since the filter employs a linearization method based on the first-order truncated Taylor series.

The UKF was developed in order to overcome the limitations of the EKF due to first-order linearization of the non-linear systems and provides derivative-free higher-order approximations with Gaussian distribution rather than arbitrary non-linear functions [28-35]. In comparison to the UKF, the EKF is difficult to implement and tune, and is mostly suboptimal for non-linear systems [28]. The UKF is more accurate and robust when adopted for navigation applications and it provided much better convergence characteristics [29]. The UKF uses a process called Unscented Transformation (UT), which evaluates the statistics of a non-linear transformed random variable $[28,29]$. A number of 'Sigma Points' are generated and propagated in order to gather information on the state vector. Different variations of the UKF were implemented, one of which is the Square RootUnscented Kalman Filter (SR-UKF) that was first proposed by J.E. Potter because the UKF performance was not reliable in 36-bit floating point arithmetic, for the Apollo flight computer, it required a KF with 15-bit fixed-point arithmetic.

Page 2 of 10 
The SR-UKF provides a robust solution with no degradation in performance compared to the UKF with the additional benefit of exhibiting reduced computational burden. The SR-UKF also prevents numerical instabilities that are inherently present in the UKF algorithm [24]. The SR-UKF also demonstrates increased numerical robustness and a reduction in computational cost [33]. A number of variants for the original KF have been developed and are illustrated in Figure 2.

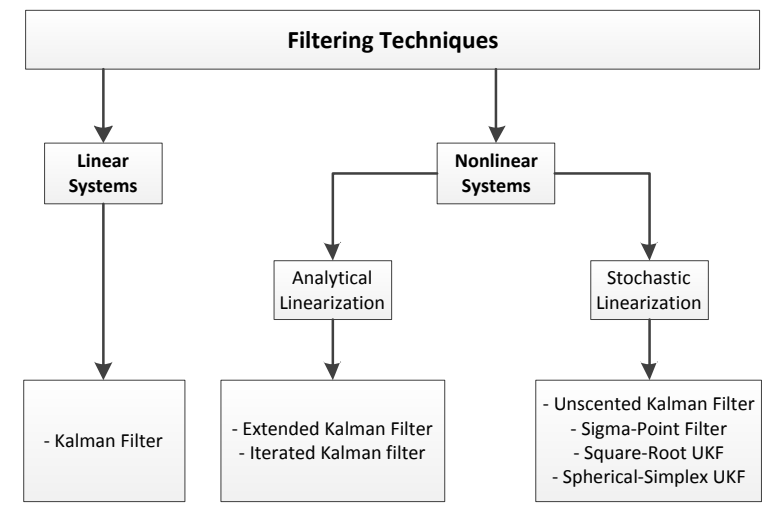

Figure 2. Kalman filter classification.

Our previous research activities [13-23] presented the various sensor choices for data fusion and the overall implementation of the VBN/IMU/GNSS/ADM (VIGA) NGS and UKF based VIGA (U-VIGA) architecture. In this paper, we propose an integrated NGS approach using SR-UKF employing three state-of-the-art physical sensors: MEMS-IMU, GNSS and VBN sensors, as well as augmentation from ADM [4-8 and 20-22].

\section{Square Root-Unscented Kalman Filter}

In its original form, the KF acts as an optimal algorithm for information processing in systems with a linear model of dynamics and measurement [36]. The KF cannot be used for non-linear systems. Therefore, other methods of estimation have been formulated and implemented for non-linear systems. Variations to the original linear Gaussian KF include non-linear Gaussian approximation filters such as the EKF, UKF and Central Difference Kalman Filter (CDKF) and sequential Monte Carlo methods such as the Particle Filter (PF). The basic framework for discrete-time state estimation of non-linear dynamic systems is presented in the following form:

$$
\begin{gathered}
x_{k+1}=f\left(x_{k}\right)+Q_{k} \\
y_{k}=h\left(x_{k}\right)+R_{k}
\end{gathered}
$$

where $x_{k}$ is discrete state vector (i.e., the unobserved state of the system), $y_{k}$ is discrete measurement vector (i.e., observed measurement signal), $k$ represents time, and $f$ and $h$ are functions on the state vector. The process noise $Q_{k}$ drives the dynamic system, and the observation noise is given by $R_{k}$. The UKF involves the recursive application of this "sampling" approach to the state-space equations [33]. A flow diagram for the UKF process is illustrated in Figure 3.

Page 3 of 10

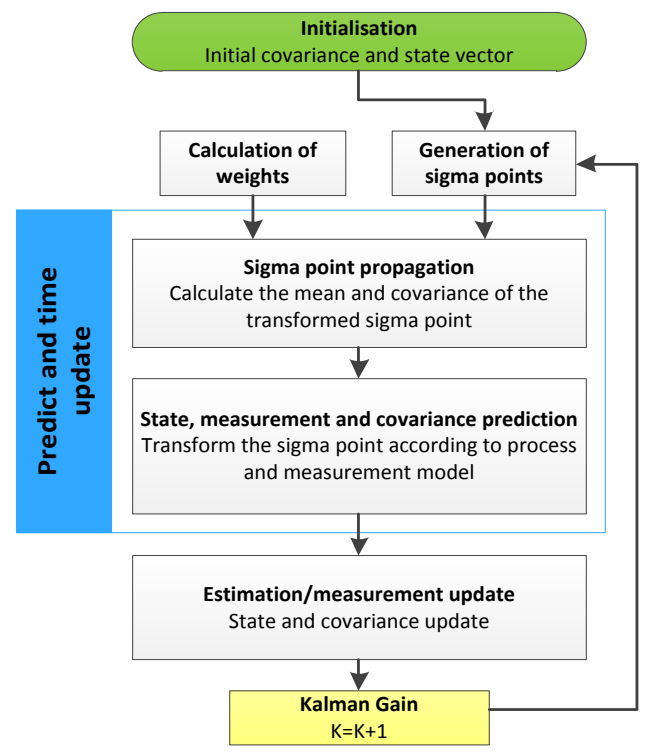

Figure 3. High-level operation of the UKF process.

The Unscented Transform (UT) process is a mathematical function that is used to estimate a given non-linear transformation using probability distribution that is characterized only in terms of a finite set of statistics. The most common use of the UT is in the non-linear projection of mean and covariance estimates in the context of nonlinear extensions of the KF [29]. The UT, especially as part of the $\mathrm{UKF}$, has largely replaced the EKF in many non-linear filtering and control applications, including underwater [37], ground and air navigation [36], and spacecraft [35] applications. The figure below depicts the UT process of the UKF and SR-UKF. The UT process is illustrated in Figure 4.

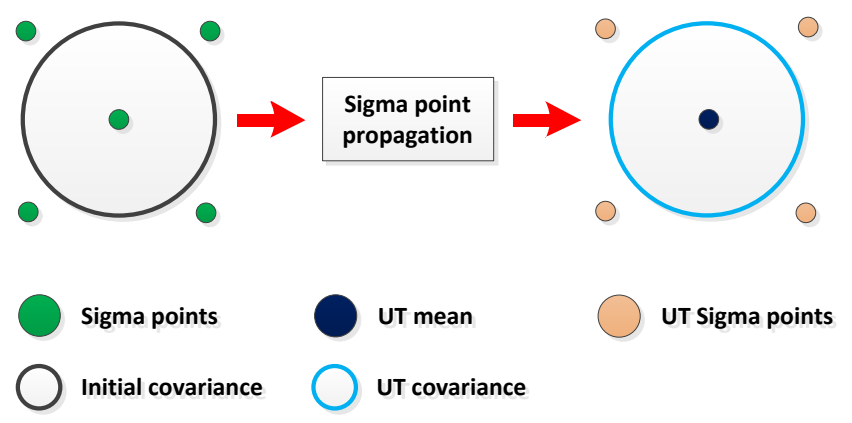

Figure 4. Unscented Transformation (UT) process.

The accuracy provided by the UKF algorithm can be improved by implementing the square root version. The UKF calculates new sigma points in every time update and hence it requires the computation of a matrix square-root of the state covariance. As in the original UKF, the filter is initialised by calculating the matrix square-root of the state covariance once via a Cholesky factorization [27]. However, the 
propagated and updated Cholesky factor is then used in subsequent iterations directly from the sigma points. During time-update, Cholesky factor given by $S$ is calculated using a QR decomposition of the compound matrix containing the weighted propagated sigma points and the square root of the additive process noise covariance. The subsequent Cholesky update (or downdate) updates the downdate process is required, because there is a possibility the weight $W_{0}^{(c)}$ may be negative [24]. Additive initialization of the SR-UKF begins with the calculation of the state mean and covariance values:

$$
\begin{gathered}
\hat{x}_{0}=m\left[x_{0}\right] \\
S_{0}=\operatorname{chol}\left\{m\left[\left(x_{0}-\hat{x}_{0}\right)\left(x_{0}-\hat{x}_{0}\right)^{T}\right]\right\}
\end{gathered}
$$

where $\hat{x}_{0}$ is the initial state vector estimate, $m$ is the mean, $x_{0}$ is the initial state vector which incorporates the initial state of the ADM, for this step a best estimate can be used if no initial state information is provided. $S_{0}$ is the initial state covariance matrix and $T$ is the transposition of the matrix, The sigma points are then calculated from the non-augmented states, so then they can be transformed through the non-linear dynamics of the process model [24]:

$$
\chi_{k-1}=\left[\begin{array}{lll}
\hat{x}_{k-1} & \hat{x}_{k-1}+\gamma S_{k} & \hat{x}_{k-1}-\gamma S_{k}
\end{array}\right]
$$

where $S$ computes the diagonal of state covariance matrix and results in the lower triangular matrix of the state covariance matrix $S_{0}$ and $\gamma$ is the control parameter of the dispersion distance from the mean estimate in the computation of the sigma point matrix. After the sigma points are calculated, a time update for each time step $k$ is performed and is given by [24]:

$$
\begin{gathered}
\chi_{k-1}=f\left[\chi_{k-1}\right] \\
\hat{x}_{k}^{-}=\sum_{i=0}^{2 n} W_{i}^{(m)} \chi_{k \mid i}^{*} \\
S_{x_{k}}^{-}=q r\left\{\left[\sqrt{W_{1}^{(C)}}\left(\chi_{k \mid 1: 2 n}^{*}-\hat{x}_{k}^{-}\right) S_{w}\right]\right\} \\
S_{\bar{x}_{k}}^{-}=\text {cholupdate }\left\{S_{\bar{x}_{k}}^{-} \chi_{k \mid 0}^{*}-\hat{x}_{k}^{-}, W_{0}^{(C)}\right\} \\
\chi_{k}=\left[\chi_{k}^{*} \chi_{k \mid 0}^{*}+\gamma S_{w} \chi_{k \mid 0}^{*}+\gamma S_{w}\right] \\
y_{k \mid i}=\mathrm{h}\left[\chi_{k \mid i}\right] \\
\hat{y}_{k}^{-}=\sum_{i=0}^{2 n} W_{i}^{(m)} y_{k \mid i}
\end{gathered}
$$

Following the time update (i.e., prediction equations) where the new mean and covariance values are calculated for the process function, the measurement function (i.e., correction equations) mean and covariance are then calculated [24].

$$
\begin{gathered}
S_{y_{k}}=q r\left\{\left[\sqrt{W_{1}^{(C)}}\left[y_{k \mid 1: 2 n}-\hat{y}_{k}\right] S_{v}\right]\right\} \\
S_{y_{k}}=\text { cholupdate }\left\{S_{\left.y_{k}, y_{k \mid 0}-\hat{y}_{k}^{-}, W_{0}^{(C)}\right\}}\right. \\
P_{x y_{k}}=\sum_{i=0}^{2 n} W_{i}^{(C)}\left[\chi_{k \mid i}-\hat{x}_{k}^{-}\right]\left[y_{k \mid i}-\hat{y}_{k}^{-}\right]^{T}
\end{gathered}
$$

The last step in the SR-UKF algorithm employs the characteristic KF equations (i.e., Kalman gain and update equations).

$$
\begin{gathered}
\mathcal{K}_{k}=\left[P_{x y_{k}} S_{y_{k}}^{T}\right] / S_{y_{k}} \\
\hat{x}_{k}=\hat{x}_{k}^{-}+\mathcal{K}_{k}\left(y_{k}-\hat{y}_{k}^{-}\right) \\
U=\mathcal{K}_{k} S_{y_{k}} \\
S_{k}=\text { cholupdate }\left\{S_{x_{k}}^{-}, U,-1\right\}
\end{gathered}
$$

The Kalman gain $\mathcal{K}_{k}$ is used to quantify the influence of new information present in the innovation vector on the estimation of the state vector and can be considered as a weight factor. The posterior measurement update of the Cholesky factor of the state covariance is calculated in equation 19 by applying $n$ (i.e., state size) sequential Cholesky downdates to $S_{k}$. The downdate vectors are the columns of $\mathcal{K}_{k} S_{\bar{y}_{k}}$. This replaces the posterior update of $P_{k}$ in with $S_{k}$ in the last covariance update.

\section{Fuzzy/PID Controller}

In order to design the control system, a hybrid approach was adopted allowing the controller to take advantage of the proposed integrated navigation sensors during all phases of flight including precision approach and landing. Therefore, fuzzy logic and Proportional/Integral/Derivative (PID) control strategies were adopted for controlling the RPAS. PID is the simplest type of linear controller and is used in most RPAS control systems. The PID control law consists of three basic feedback signals, namely proportional, integral and derivative with gains $\mathrm{K}_{\mathrm{p}}, \mathrm{K}_{\mathrm{i}}$ and $\mathrm{K}_{\mathrm{d}}$ respectively. The gains affect the system as follows:

- $\quad P$ term: Increasing $K_{p}$ speeds up the response of the system

- I term: The integral controller eliminates steady state error and

- D term: The derivative controller increases the stability of the system and has no effect on the steady state error.

Fuzzy logic is a form of multi-value logic based on a representation of knowledge and reasoning of a human operator. In contrast to conventional PID controllers, Fuzzy Logic Controllers (FLC) do not require a model of the system. Therefore, it can be applied to nonlinear systems or various ill-defined processes for which it is difficult to model the dynamics. The process consists of four components: fuzzification, fuzzy rule base, inference engine and defuzzification. Fuzzification refers to transforming a crisp set into a fuzzy set using linguistic terms. A Membership Function (MF) is defined as a curve that classifies how each point in the input space is mapped to a membership value (or a degree of membership) between 0 and 1 [38]. An example is provided in Figure. 5.

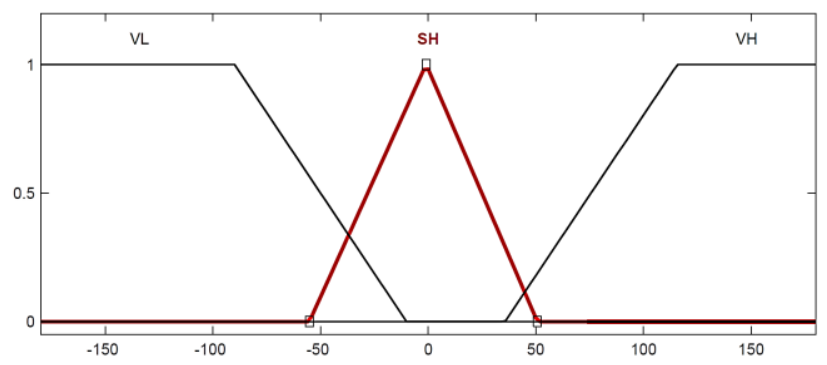

Figure 5. Input fuzzy sets and their membership functions.

The second component, that is the Fuzzy rule base, forms the main part of fuzzy logic. It is based on if-then rules that tell the controller how to react to the inputs. Defuzzification is the method to obtain the output from the controller. It converts the output fuzzy set value to a crisp set using its membership functions. The RPAS controller design was approached by decoupled dynamic models of the aircraft resulting in two complimentary controllers, one for lateral motion and one for longitudinal motion. The functional architecture of the controller is illustrated in Figure. 6. 


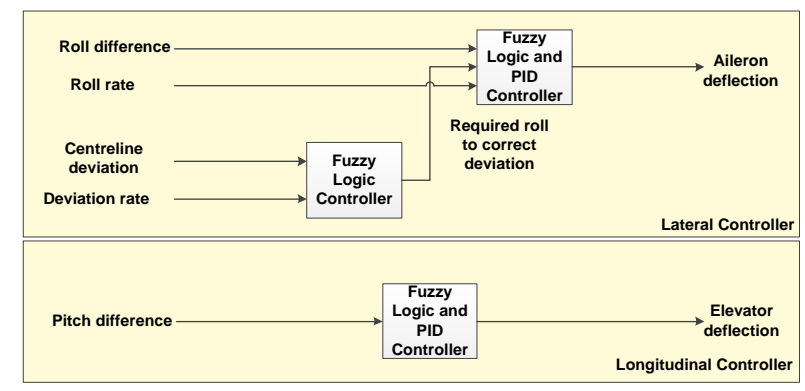

Figure 6. Functional architecture of the controller.

Before initiating the controller design, the open-loop response of the system was first tested. In open-loop flight, the control inputs were set to a fixed value without any feedback from the aircraft states. It is observed that the RPAS is unstable in this condition and settles in a constant bank turn and pitch angle. The pitch angle open-loop response is shown in Figure 7. This is due to the propulsion system, which causes an unbalanced roll moment and excites the spiral mode.

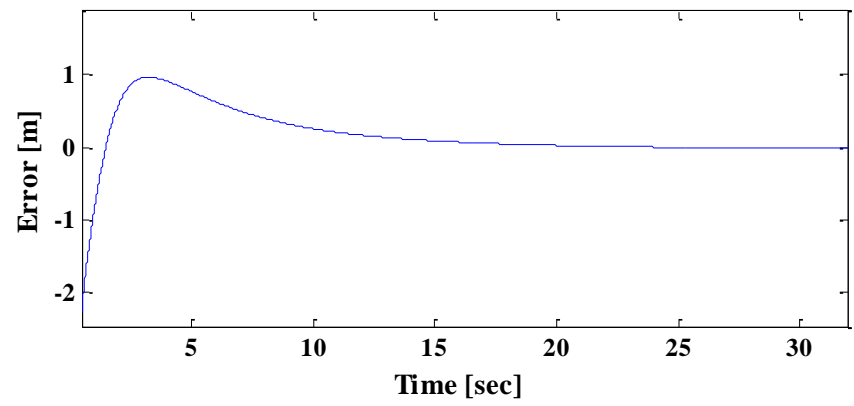

Figure 7. Pitch angle open-loop response (spiral mode).

The lateral controller was first designed to stabilise the lateral dynamics of the RPAS. This was followed by the longitudinal controller to control the pitch angle. The overall design was then adapted to perform servoing using the information from the VBN sensors and integrated navigation systems. Triangular and trapezoidal membership functions were used for the membership functions due to their simplicity and ease of implementation. The membership functions which gave the best results for the roll and pitch responses were selected. Linguistic variables were used to define the fuzzy sets of inputs and the outputs of the controller. The fuzzy sets and the range of the inputs and outputs are shown in Tables 1 and 2, where $\mathrm{VN}=$ Very Negative, $\mathrm{VP}=$ Very Positive, $\mathrm{VH}=$ Very High, $\mathrm{VL}=$ Very Low, $\mathrm{SN}=$ Slightly Negative, $\mathrm{SP}=$ Slightly Positive, $\mathrm{SH}=$ Slightly High, $\mathrm{SL}=$ Slightly Low, $\mathrm{Z}=$ Zero.

Table 1. Fuzzy sets and range of inputs.

\begin{tabular}{ccc}
\hline Input Variable & Fuzzy Set & Range \\
\hline Roll error & VN, SN, Z, SP, VP & $-180^{\circ}$ to $180^{\circ}$ \\
Roll rate & VN, SN, Z, SP, VP & $-40^{\circ} / \mathrm{s}$ to $40^{\circ} / \mathrm{s}$ \\
Pitch error & VL, SL, Z, SH, VH & $-90^{\circ}$ to $90^{\circ}$ \\
Deviation & VN, SN, Z, SP, VP & -512 to 512 pixels \\
Deviation rate & VN, SN, Z, SP, VP & -600 to 600 pixels/s \\
\hline
\end{tabular}

Page 5 of 10
Table 2. Fuzzy sets and range of outputs.

\begin{tabular}{ccc}
\hline Output Variable & Fuzzy Set & Range \\
\hline Aileron deflection & $\mathrm{VN}, \mathrm{SN}, \mathrm{Z}, \mathrm{SP}, \mathrm{VP}$ & $-60^{\circ}$ to $60^{\circ}$ \\
Elevator deflection & $\mathrm{VN}, \mathrm{SN}, \mathrm{Z}, \mathrm{SP}, \mathrm{VP}$ & $-60^{\circ}$ to $60^{\circ}$ \\
$\begin{array}{c}\text { Required roll to } \\
\text { correct deviation }\end{array}$ & $\mathrm{VN}, \mathrm{SN}, \mathrm{Z}, \mathrm{SP}, \mathrm{VP}$ & $-60^{\circ}$ to $60^{\circ}$ \\
\hline
\end{tabular}

The lateral controller design was designed with the aim of stabilising the roll of the aircraft during the landing phase. This was required to maintain zero roll during touchdown at the centre of the runway so as to avoid wing-strike on the runway. It also controlled the position of the aircraft with respect to the centreline of the runway. The design process of the longitudinal controller followed the same methodology as that of the lateral controller. A derivative gain was used instead of pitch rates. The fuzzy rules used for the longitudinal controller are given below:

- If (Pitch is Z) then (Elevator_Deflection is Z)

- If (Pitch is SH) then (Elevator_Deflection is SP)

- If (Pitch is SL) then (Elevator_Deflection is SN)

- If (Pitch is VH) then (Elevator_Deflection is VN)

- If (Pitch is VL) then (Elevator_Deflection is VP)

The pitch angle close-loop response of the controller is shown in Figure 8 .

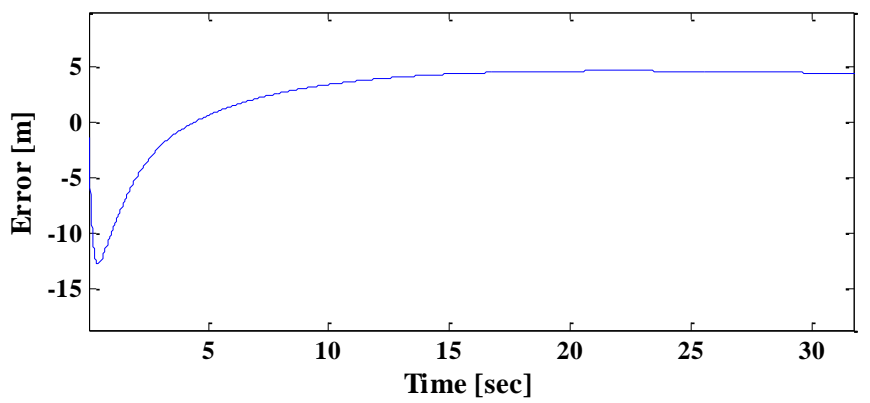

Figure 8. Pitch angle close-loop response with controller.

The results show that the pitch and roll converge rapidly towards the required value of zero after a short initial instability. The simulation showed that the controller is able to correct the attitude disturbances caused by moderate to high wind speeds. However, it was observed that the aircraft became unstable with lateral wind speeds exceeding $20 \mathrm{~m} / \mathrm{s}$. The implementation of UKF based NGS architecture provided better results when the disturbances existed.

\section{Aircraft Dynamics Model}

With reference to the implementation of the ADM, six Degrees-ofFreedom (6-DOF) geodetic non-linear equations force and moment equations are listed below, describing the motion of the aircraft in the body frame $G$ with:

- A velocity $V=[u v w]^{T}$ with regards to the inertial frame, expressed in the body frame $G$

- An angular rates $w=[p q r]$

where $u$ is the axial velocity, $v$ is the lateral velocity and $w$ is the normal velocity, $p$ is the roll rate, $q$ is the pitch rate and $r$ is the yaw rate. The equations describing the forces applied on the aircraft are 
described below. Gravitational forces act on the gravity center of the aircraft and are given by:

$$
\begin{gathered}
\left(F_{x}\right)_{\text {gravity }}=-m g \sin \vartheta \\
\left(F_{y}\right)_{\text {gravity }}=m g \cos \vartheta \sin \varphi \\
\left(F_{z}\right)_{\text {gravity }}=m g \cos \vartheta \cos \varphi
\end{gathered}
$$

where $\vartheta$ is pitch, $\varphi$ is roll, $\psi$ is yaw angle, $\mathrm{G}$ is acceleration due to gravity and $\mathrm{M}$ is mass of the RPAS. Similarly, propulsion forces are given by:

$$
\begin{gathered}
\left(F_{x}\right)_{\text {engine }}=T \cos \epsilon \\
\left(F_{y}\right)_{\text {engine }}=0 \\
\left(F_{z}\right)_{\text {engine }}=-T \sin \epsilon
\end{gathered}
$$

where $T$ is thrust supplied by engines, $\epsilon$ is angle between the $X_{b}$ - axis and the mounted axis of the engines. The aerodynamic forces acting on the $\mathrm{x}, \mathrm{y}$ and $\mathrm{z}$ axis are denoted as $X, Y$ and $Z$ respectively. The set of forces equations are given by:

$$
\begin{gathered}
m(\dot{u}+q w-r v)+u * \dot{m}=X-m g \sin \vartheta+T \cos \epsilon \\
m(\dot{v}+r u-p w)+v * \dot{m}=Y+m g \cos \vartheta \sin \varphi \\
m(\dot{w}+p w-q u)+w * \dot{m}=Z+m g \cos \vartheta \cos \varphi-T \sin \epsilon
\end{gathered}
$$

The kinematic moments are expressed as:

$$
\begin{gathered}
H_{x}=p I_{x}-q I_{x y}-r I_{x z} \\
H_{y}=-p I_{x y}+q I_{y}-r I_{y z} \\
H_{z}=-p I_{x z}-q I_{y z}+r I_{z}
\end{gathered}
$$

where:

$$
\begin{gathered}
I_{x}=\iiint y^{2}+z^{2} d m ; I_{y}=\iiint x^{2}+z^{2} d m \\
I_{x}=\iiint x^{2}+y^{2} d m ; I_{x y}=\iiint x y d m \\
I_{y z}=\iiint y z d m ; I_{x z}=\iiint x z d m
\end{gathered}
$$

The moment applied on the aircraft in the $\mathrm{x}, \mathrm{y}$ and $\mathrm{z}$ axis are denotes as $\mathrm{L}, \mathrm{M}$ and $\mathrm{N}$ respectively. The set of moment equations are given by:

$$
\begin{array}{r}
I_{x} \dot{p}+I_{x z} \dot{r}+\left(I_{z}-I_{y}\right) q r+I_{x z} q p=L \\
I_{y} \dot{q}+\left(I_{x}-I_{x}\right) p r+I_{x z}\left(r^{2}-p^{2}\right)=M \\
I_{z} \dot{r}+I_{x z} \dot{p}+\left(I_{y}-I_{x}\right) q p-I_{x z} q r=N
\end{array}
$$

The ADM used for simulation purposes is modeled according to the following assumptions:

- The Earth model chosen is the WGS-84 model, which approximates the Earth as an ellipsoid

- The atmosphere is considered at rest relatively to the Earth, and the atmospheric model is the Standard Atmosphere (SA), defining temperature, pressure and density as a function of altitude

- Aircraft engine is rigidly mounted on the vehicle body and the aircraft mass is located in the aircraft centre of gravity and hence the mass is varying only as a result of fuel consumption

- $\quad$ All wind effects and sideslip are neglecting

- Uniform gravity and the geodetic coordinate system of reference is World Geodetic System of year 1984 (WGS 84)

The uncertainties in the aerodynamic parameters are the primary source of errors in the model resulting from the use of the ADM. The accuracy of these parameters depends on the source of the data, which are theoretical computations, wind tunnel experiments and flight tests.

\section{Multi-Sensor Navigation and Guidance System Architectures}

The two MSDF based NGS architectures compared are the UKF based (U-VIGA) and SR-U-VIGA based system. The U-VIGA architecture illustrated in Figure 9 uses VBN at $20 \mathrm{~Hz}$ and Global Positioning System (GPS) at $1 \mathrm{~Hz}$ to augment the MEMS-IMU running at $100 \mathrm{~Hz}$. This architecture includes ADM (computations performed at $100 \mathrm{~Hz}$ ) to provide attitude channel augmentation. The sensor measurements are handled by a sensor processing and data sorting block. The data sorting algorithm is based on Boolean Decision Logics (BDL), which allow automatic selection of the sensor data based on pre-defined priority criteria. The sorted data is then fed to an UKF to obtain the best estimate values. The INS position and velocity are compared with the GPS position and velocity to form the measurement input of the data fusion block containing the UKF. The attitude data provided by the ADM and the INS are compared to feed the UKF at $100 \mathrm{~Hz}$, and the attitude data provided by the VBN sensors and INS are compared at $20 \mathrm{~Hz}$ and form the inputs to the UKF and SR-UKF.

Both UKF and SR-UKF provides estimates of Position, Velocity and Attitude (PVA) errors, which are then removed from the sensor measurements to obtain the corrected PVA states. An additional UKF is used to process the ADM navigation solution. IN contrast to the previously implemented EKF based E-VIGA system, the ADM operates in parallel to the centralized UKF and acts as a separate subsystem. The processing of the ADM virtual sensor measurements leads to reduction of the overall position and attitude error budget and importantly considerable reduction in the ADM re-initialization time. PVA measurements are obtained as state vectors from both the centralised UKF and the Aircraft Dynamics Filter (ADF) (i.e., processed $\mathrm{ADM}$ ). These measurements are then fed into an error analysis module in which the measurement values of the two UKF are compared. The error analysis block includes the primary sensors (GNSS, INS and VBN) and it is used to compare the VIG error values with the virtual sensor (ADM) error values to obtain the corrected PVA states. The SR-U-VIGA architecture is illustrated in Figure 10. 


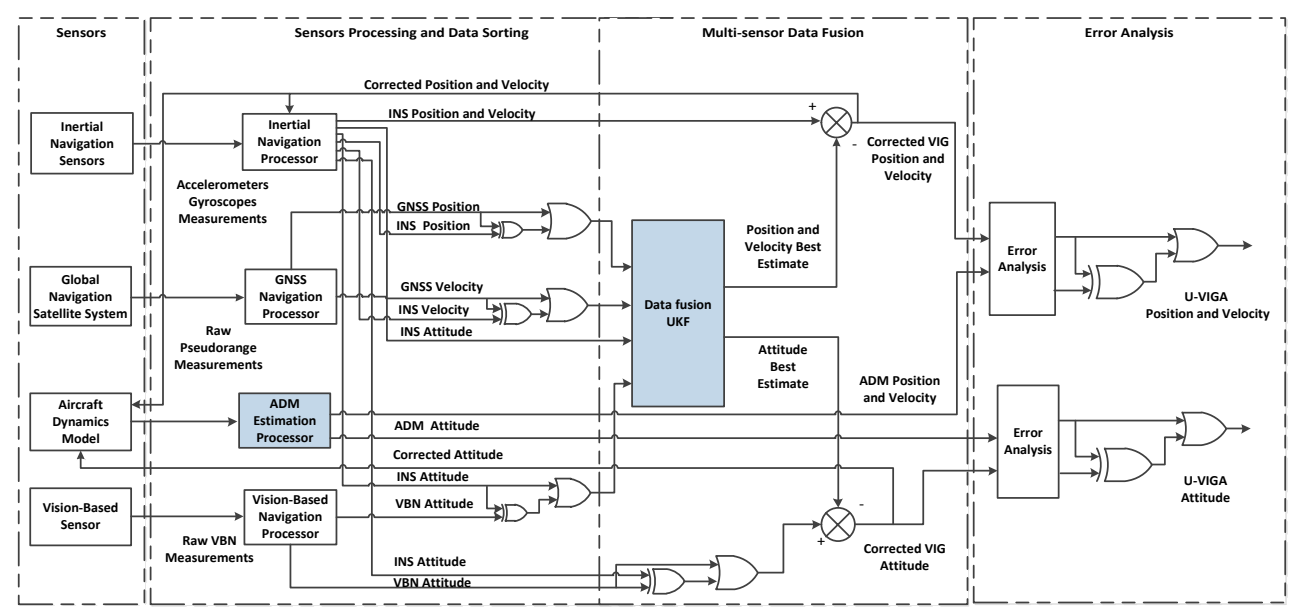

Figure 9. U-VIGA architecture.

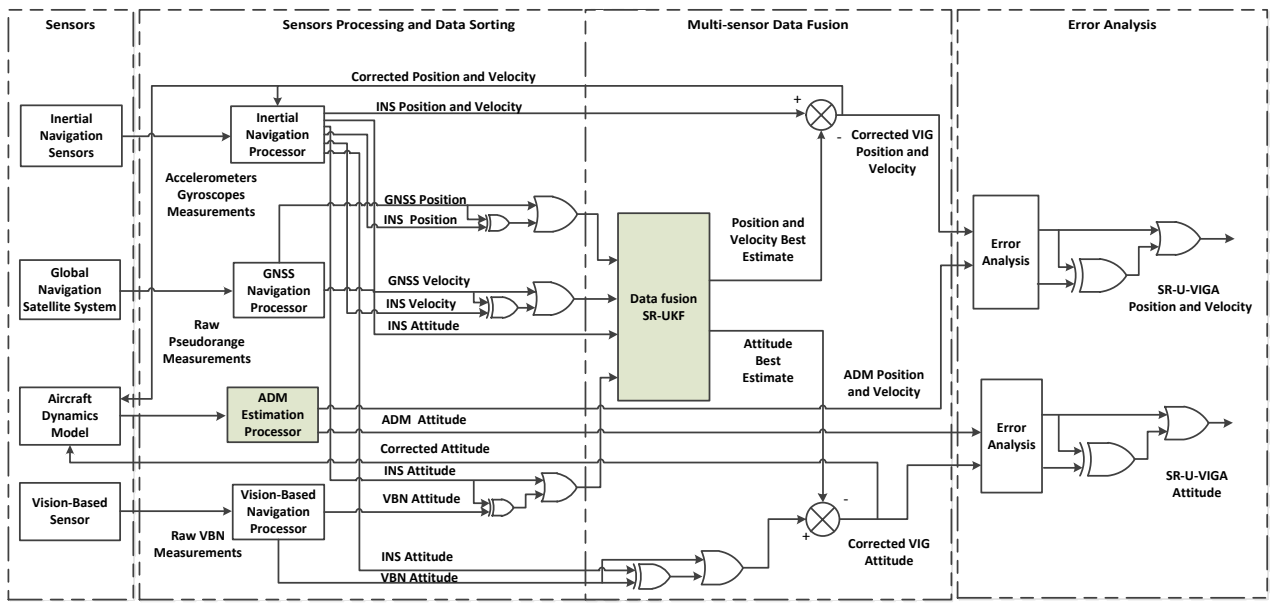

Figure 10. SR-U-VIGA architecture.

\section{Simulation Case Study}

A detailed case study was performed in a high dynamics RPAS environment, employing a 6-DoF model of the AEROSONDE RPAS as the reference ADM. The corresponding E-VIGA and U-VIGA integrated navigation modes were simulated using MATLAB ${ }^{\mathrm{TM}}$ in an appropriate sequence of flight manoeuvres representative of the AEROSONDE RPAS operational flight envelope. The duration of the simulation is 950 seconds covering twelve flight legs from starting point to destination. The 3D trajectory plot of the flight profiles of the AEROSONDE RPAS is illustrated in Figure 11. 


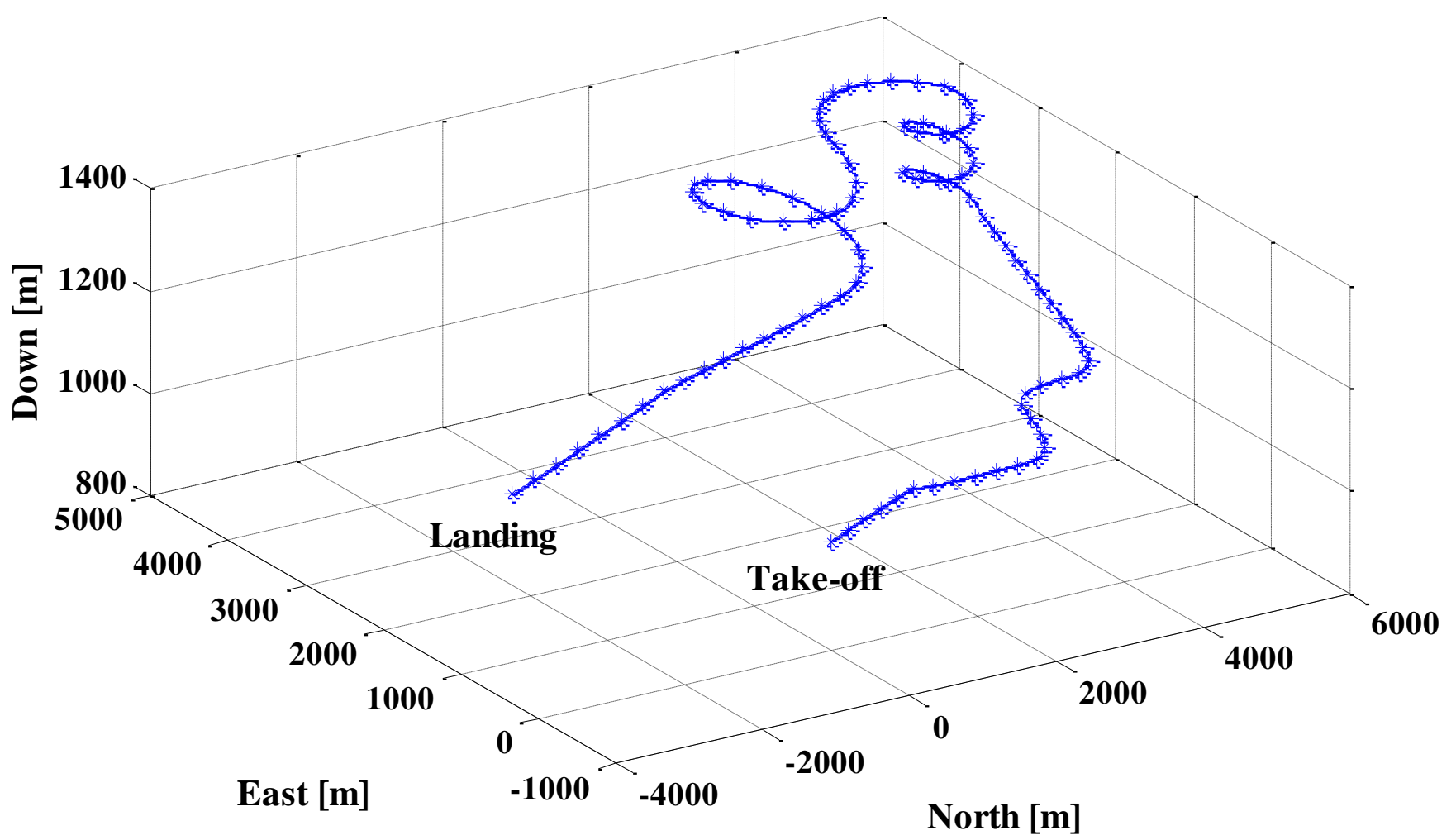

Figure 11. 3D trajectory plot of RPAS flight profile.

The best estimates of PVA for the two NGS architectures are obtained and the associated error statistics (mean, $\mu$ and standard deviation, $\sigma$ ) are calculated. Tables 3 and 4 list the position and attitude error statistics of the two NGS architectures respectively. The E-VIGA NGS system is prone to rapid divergence and its optimal time for re-initialisation is in the order of 20 seconds. The U-VIGA NGS and SR-U-VIGA systems show considerable improvement in the horizontal and vertical positions. By applying an UKF to process the ADM measurements, the navigational solution is corrected and becomes suitable for an extended time of operation. Compared with the E-VIGA solution, a significant improvement of the solution validity time is obtained with the U-VIGA and SR-U-VIGA systems. In particular, the lateral position validity time before the solution exceeds the RNP 1 threshold in the climb phase is $215 \mathrm{sec}$ and, in the final approach phase, the ADM solution exceeds the CAT I, CAT II and CAT III limits at $149 \mathrm{sec}, 130 \mathrm{sec}$ and $44 \mathrm{sec}$ respectively (the EVIGA was compliant with RNP 1 threshold up to $98 \mathrm{sec}$, CAT I up to $79 \mathrm{sec}$, CAT II up to $50 \mathrm{sec}$ and CAT III up to $36 \mathrm{sec}$ ). The vertical position validity time before the solution exceeds the RNP 1 threshold in the climb phase is $194 \mathrm{sec}$ in the case of SR-U-VIGA. Furthermore, CAT II and CAT III requirements were satisfied up to $60 \mathrm{sec}$ and CAT I requirements up to $108 \mathrm{sec}$. The E-VIGA was compliant with RNP 1 threshold up to $89 \mathrm{sec}$, CAT I up to $56 \mathrm{sec}$, CAT II and CAT III up to $21 \mathrm{sec}$ in the case of vertical position. Based on the requirmeents set by ICAO, the obtained results are in line with CAT II precision approach requirements. The comparison of lateral and vertical guidance validity times of the EKF, UKF and SR-UKF filters is provided in Table 5.
Table 3. Position error statistics.

\begin{tabular}{|c|c|c|c|c|c|c|}
\hline \multirow{2}{*}{$\begin{array}{c}\text { NGS } \\
\text { Architecture }\end{array}$} & \multicolumn{2}{|c|}{$\begin{array}{c}\text { North } \\
\text { Position [m] }\end{array}$} & \multicolumn{2}{c|}{$\begin{array}{c}\text { East } \\
\text { Position [m] }\end{array}$} & \multicolumn{2}{c|}{$\begin{array}{c}\text { Down } \\
\text { Position [m] }\end{array}$} \\
\cline { 2 - 7 } & $\mu$ & $\sigma$ & $\mu$ & $\sigma$ & $\mu$ & $\sigma$ \\
\hline U-VIGA & 0.34 & 2.00 & -0.49 & 1.94 & 0.17 & 2.45 \\
\hline SR-VIGA & 0.35 & 2.00 & -0.40 & 1.97 & 0.21 & 2.25 \\
\hline
\end{tabular}

Table 4. Attitude error statistics.

\begin{tabular}{|c|c|c|c|c|c|c|}
\hline \multirow{2}{*}{$\begin{array}{c}\text { NGS } \\
\text { Architecture }\end{array}$} & \multicolumn{2}{|c|}{$\begin{array}{c}\text { Pitch }(\theta) \\
\text { [degrees] }\end{array}$} & \multicolumn{2}{c|}{$\begin{array}{c}\text { Roll }(\phi) \\
\text { [degrees] }\end{array}$} & \multicolumn{2}{c|}{$\begin{array}{c}\text { Heading }(\psi) \\
\text { [degrees] }\end{array}$} \\
\cline { 2 - 7 } & $\mu$ & $\sigma$ & $\mu$ & $\sigma$ & $\mu$ & $\sigma$ \\
\hline $\begin{array}{c}\text { U-VIGA } \\
\text { x10^-3 }\end{array}$ & 5.5 & 40.7 & -6.9 & 314.0 & -1.7 & 44.9 \\
\hline $\begin{array}{c}\text { SR-VIGA } \\
\text { x10^-3 }\end{array}$ & 5.4 & 40.0 & -6.5 & 311.7 & 2.0 & 47.7 \\
\hline
\end{tabular}


Table 5. E-VIGA, U-VIGA and SR-U-VIGA ADM lateral and vertical guidance validity times.

\begin{tabular}{|c|c|c|c|c|}
\hline \multirow{3}{*}{$\begin{array}{l}\text { Accuracy } \\
\text { threshold }\end{array}$} & \multicolumn{4}{|c|}{$\mathrm{ADM}$ validity time $[\mathrm{sec}]$} \\
\hline & \multicolumn{2}{|c|}{ Lateral Position } & \multicolumn{2}{|c|}{ Vertical Position } \\
\hline & E-VIGA & $\begin{array}{c}\text { U-VIGA / } \\
\text { SR-U-VIGA }\end{array}$ & E-VIGA & $\begin{array}{c}\text { U-VIGA / } \\
\text { SR-U-VIGA }\end{array}$ \\
\hline RNP 1 & 98 & 215 & 89 & 194 \\
\hline CAT I & 79 & 149 & 56 & 108 \\
\hline CAT II & 50 & 130 & \multirow{2}{*}{21} & \multirow{2}{*}{60} \\
\hline CAT III & 36 & 44 & & \\
\hline
\end{tabular}

The computational time of the filters are obtained from a single integration step. The results show the EKF takes $0.1122 \mathrm{sec}$, the UKF $0.0013 \mathrm{sec}$ and the SR-UKF $0.00068 \mathrm{sec}$ for computation and it is observed that the SR-UKF provides the most efficient performance with significantly reduced computation cost. The simulations were executed on the Windows 7 Enterprise platform (64-bit operating system) supported by the Intel(R) Core2 Duo E8500 CPU with clock speed $3.17 \mathrm{GHz}$ and 4.0 GB RAM. The obatained computation times are tabulated in Table 6 .

Table 6. Comparison of computation times.

\begin{tabular}{|c|c|c|c|}
\hline $\begin{array}{c}\text { NGS } \\
\text { Architecture }\end{array}$ & E-VIGA & U-VIGA & SR-U-VIGA \\
\hline $\begin{array}{c}\text { Computation } \\
\text { Time [sec] }\end{array}$ & 0.1122 & 0.0013 & 0.00068 \\
\hline
\end{tabular}

\section{Conclusions and Future Work}

A low-cost and low-weight/volume integrated state-of-the-art NGS exploring an innovative MSDF architecture was developed and the system is processed with estimation techniques adopted for non-linear systems. This paper presents the results obtained from implementing a SR-UKF based NGS in an innovative low-cost MSDF architecture. Various sensors were considered for the NGS design including GNSS and MEMS-IMU, with augmentation from ADM and VBN sensors. The SR-U-VIGA was developed based on a traditional UKF based UVIGA system. The SR-U-VIGA system shows superior performance to the U-VIGA architecture for real time applications, where computational efficiency is the key factor for the NGS performance.The SR-U-VIGA system employed an additional UKF for processing the ADM navigation solution (i.e., attitude channel data) and thus also increased the ADM solution validity time. When compared with the E-VIGA system used in previous research activities, the U-VIGA and SR-U-VIGA systems showed an improvement of accuracy in the position and attitude measurements in addition to an increased ADM validity time. Additionally, the integration schemes achieved horizontal/vertical position accuracies in line with precision approach requirements. The novel Fuzzy/PID controller implemented for the NGS design was also described. Future research will address uncertainty analysis and possible synergies of the U-VIGA and SR-U-VIGA architectures with GNSS Avionics Based Integrity Augmentation (ABIA) systems [39].

\section{References}

1. Lacher, A., et al., "Airspace Integration Alternatives for Unmanned Aircraft," Centre for Advanced Aviation System Development, The MITRE Corporation, 2010.

2. DeGarmo, M.T. and Nelson, G.M., "Prospective Unmanned Aerial Vehicle Operations in the Future National Airspace System," AIAA $4^{\text {th }}$ Aviation Technology, Integration and Operations (ATIO) Forum, 2004.

3. DeGarmo, M.T., "Issues Concerning Integration of Unmanned Aerial Vehicles in Civil Airspace," The MITRE Corporation, McLean, Virginia, 2004.

4. Sabatini, R., Ramasamy, S., Cappello F., and Gardi, A., "RPAS Navigation and Guidance Systems based on GNSS and other Low-Cost Sensors," Fourth Australasian Unmanned Systems Conference 2014 (ACUS 2014), Melbourne, Australia, doi: 10.13140/2.1.3792.8008.

5. SESAR, "Modernising the European Sky," Brussels, Belgium: SESAR Consortium, 2011.

6. ICAO, "Doc 9750 - Global Air Navigation Capacity \& Efficiency Plan 2013-2028," 4 edition, Montreal, Quebec, Canada: International Civil Aviation Organization (ICAO), 2014.

7. SESAR and the Environment. Brussels, Belgium: European Commission, EU, 2014.

8. Sabatini, R., Gardi, A., Ramasamy, S., Kistan, T., and Marino, M., "Novel ATM and Avionic Systems for Environmentally Sustainable Aviation Conference: Practical Responses to Climate Change (PRCC) 2014," Engineers Australia Convention 2014, Melbourne, Australia, Volume: Transport (Aviation), 2014, doi: 10.13140/2.1.1938.0808.

9. Sabatini, R., "A Roadmap for Future Aviation Research in Australia: Improving Aviation Safety, Efficiency and Environmental Sustainability," Conference: First International Symposium on Sustainable Aviation (ISSA 2015), Istanbul, Turkey, 2015, doi: 10.13140/RG.2.1.4833.2962.

10. Ramasamy, S., Sabatini, R., and Gardi, A., "Novel Flight Management System for Improved Safety and Sustainability in the CNS+A Context, Conference: Integrated Communication," Navigation and Surveillance Conference (ICNS 2015), VA, USA, 2015, doi: 10.1109/ICNSURV.2015.7121225.

11. Ramasamy, S., Gardi, A., and Sabatini, R., "A Laser Obstacle Avoidance System for Manned and Unmanned Aircraft Detectand-Avoid," $16^{\text {th }}$ Australian International Aerospace Congress (AIAC16), Melbourne, 2015, doi: 10.13140/2.1.1275.7285.

12. Sabatini, R., Moore, T., Hill, C., and Ramasamy, S., "Assessing Avionics-Based GNSS Integrity Augmentation Performance in UAS Mission-and Safety-Critical Tasks," International Conference on Unmanned Aircraft Systems (ICUAS 2015), Denver, CO, USA, 2015.

13. Ramasamy, S., Gardi, A., Liu, J., and Sabatini, R., "A Laser Obstacle Detection and Avoidance System for Manned and Unmanned Aircraft Applications," International Conference on Unmanned Aircraft Systems (ICUAS 2015), Denver, CO, USA, 2015.

14. Ramasamy, S. and Sabatini, R., "Unifying Cooperative and Non-Cooperative UAS Sense-and-Avoid," Second International Workshop on Metrology for Aerospace (MetroAeroSpace 2015), Benevento, Italy, 2015.

15. Cappello, F., Ramasamy, S., Sabatini, R., and Liu, J., "LowCost Sensors based Multi-sensor Data Fusion Techniques for RPAS Navigation and Guidance," International Conference on Unmanned Aircraft Systems (ICUAS 2015), Denver, CO, USA, 2015. 
16. Cappello, F., Sabatini, R., Ramasamy, S., and Marino, M., "Particle Filter based Multi-sensor Data Fusion Techniques for RPAS Navigation and Guidance," Second International Workshop on Metrology for Aerospace (MetroAeroSpace 2015), Benevento, Italy, 2015.

17. Ramasamy, S., and Sabatini, R., "A Unified Approach to Cooperative and Non-Cooperative Sense-and-Avoid," International Conference on Unmanned Aircraft Systems (ICUAS 2015), Denver, CO, USA, 2015.

18. Sabatini, R., Moore, T., Hill, C., and Ramasamy, S., "Evaluating GNSS Integrity Augmentation Techniques for UAS Sense-andAvoid," Second International Workshop on Metrology for Aerospace (MetroAeroSpace 2015), Benevento, Italy, 2015.

19. Cappello, F., Ramasamy, S., and Sabatini, R., "Low-Cost Sensors Data Fusion for Unmanned Aircraft Navigation and Guidance," International Journal of Science and Engineering Investigations (IJSEI), 2015. (In press)

20. Sabatini, R., Ramasamy, S., Gardi, A., and Rodríguez, L., "Low-cost Sensors Data Fusion for Small Size Unmanned Aerial Vehicles Navigation and Guidance," International Journal of Unmanned Systems Engineering, Vol. 1, No. 3, pp. 16-47, 2013, doi: 10.14323/ijuseng.2013.11.

21. Sabatini, R., Cappello, F., Ramasamy, S., Gardi, A., and Clothier R., "An Innovative Navigation and Guidance System for Small Unmanned Aircraft using Low-Cost Sensors," Journal of Aircraft Engineering and Aerospace Technology, 2015. (In press)

22. Cappello, F., Ramasamy, S., and Sabatini, R., "Multi-sensor Data Fusion Techniques for RPAS Navigation and Guidance," $16^{\text {th }}$ Australian International Aerospace Congress, 2015.

23. Cork, L.R., "Aircraft Dynamic Navigation for Unmanned Aerial Vehicles," PhD Thesis, Queensland University of Technology, 2014.

24. Lozano, R., "Unmanned Aerial Vehicles Embedded Control," John Wiley \& Sons, 2013.

25. Klein, L.A., "Sensor and data fusion: A tool for information assessment and decision making," SPIE Press, p. 51, ISBN 08194-5435-4, 2004.

26. Durrant-Whyte, H., "Multi Sensor Data Fusion," Australian Centre for Field Robotics, The University of Sydney, NSW, Australia, January, 2001.

27. AbdelAziz, O. and Hamza, B., "Comparison between extended Kalman filter and Sigma-Points Kalman filters applied to integrated navigation system INS (inertial Navigation System)/GPS (Global Positioning system) with selective Availability," IFAC Workshop, Aerospace Guidance, Navigation and Flight Control Systems, Samara, Russia, 2009.

28. Julier, S.J. and Uhlmann, J.K., "A New Extension of the Kalman Filter to Non-linear Systems," Proceedings of the International Symposium of Aerospace/Defense Sensing, Simulation and Controls, Orlando, FL, USA, pp. 182-193, 1997.

29. Julier, S.J. and Uhlmann, J.K., "Unscented Filtering and Nonlinear Estimation," Proceedings of the IEEE, vol. 92, no. 3, pp. 401-422, 2004.

30. Julier, S.J., Uhlmann, J.K. and Durrant-Whyte, H.F., "A new Approach for Filtering Non-linear Systems," Proceedings of the American Control Conference, Seattle, WA, pp. 1628-1632, 1995.

31. Lozano, J.G.C., Carrillo, L.R.G., Dzul A., and Lozano, R., "Spherical Simplex Sigma-Point Kalman Filters: A Comparison in the Inertial Navigation of a Terrestrial Vehicle," American Control Conference, Seattle, Washington, USA, 2008.

32. Julier, S., Uhlmann, J. and Durrant-Whyte, H.F., "A new Method for the Non-linear Transformation of Means and
Covariances in Filters and Estimators," IEEE Transactions on Automatic Control, vol. 45, no. 3, 2000.

33. Van Der Merwe, R. and Wan, E.A., "The Square-root Unscented Kalman Filter for State and Parameter-estimation," IEEE International Conference on Acoustics, Speech, and Signal Processing Proceedings, 2001.

34. Wan, E.A. and Van Der Merwe, R., "The unscented Kalman filter for nonlinear estimation. "Advances in Unmanned Aerial Vehicles Non-linear estimation," Proceedings of the IEEE Symposium on Adaptive Systems and Signal Processing Communications and Control, Lake Louise, Alberta, Canada, pp. 153-158, 2000.

35. Van Der Merwe, R., "Sigma-point Kalman Filters for Probabilistic Inference in Dynamic State-space Models," PhD Thesis, OGI School of Science and Engineering, Oregon Health and Science University, 2004.

36. Malinowski, M, and Kwiecień, J, "Study of the Effectiveness of Different Kalman Filtering Methods and Smoothers in Object Tracking based on Simulation Tests," University of Technology and Life Sciences Geomatics, Geodesy and Spatial Economy Department Bydgoszcz, Poland, 2014.

37. Wu, L, J, Ma., and J Tian., "Self-Adaptive Unscented Kalman Filtering for Underwater Gravity Aided Navigation," Proceedings of IEEE/ION Plans, 2010.

38. Aboul-Ella, H. and Abraham, A., "Computational Intelligence in Multimedia Processing: Recent Advances," vol. 96. Springer, 2008.

39. Sabatini, R., Moore, T., Hill, C., and Ramasamy, S., "Assessing Avionics-Based GNSS Integrity Augmentation Performance in UAS Mission-and Safety-Critical Tasks," International Conference on Unmanned Aircraft Systems (ICUAS 2015), Denver, CO, USA, June 2015.

Page 10 of 10 